\title{
Faune piscicole du bassin de la Comoé et paramètres de croissance de quelques espèces d'intérêt économique
}

\author{
Oumar SIRIMA ${ }^{1}$, Aboubacar TOGUYENI ${ }^{1,2^{*}}$ et \\ Chantal Yvette KABORE-ZOUNGRANA ${ }^{1,2}$
}

\begin{abstract}
${ }^{1}$ Laboratoire d'Etudes et de Recherches des Ressources Naturelles et des Sciences de l'Environnement (LERNSE), Université Polytechnique de Bobo-Dioulasso, 01 BP 1091 Bobo-Dioulasso 01, Burkina Faso.

${ }^{2}$ Université Polytechnique de Bobo-Dioulasso, Institut des Sciences de la Nature et de la Vie, 01 BP 1091 Bobo-Dioulasso 01, Burkina Faso.

*Auteur correspondant, E-mail: toguyenia@yahoo.fr
\end{abstract}

\section{RESUME}

L'inventaire de la faune piscicole du fleuve Comoé et de trois retenues d'eau (Bounouna, Dangouindougou, Tounoura) au Burkina Faso, a permis de recenser 40 espèces. Dans ces retenues, Oreochromis niloticus, Tilapia zillii, Sarotherodon galilaeus (Cichlidae) et Heterotis niloticus (Osteoglossidae) sont les espèces les plus abondantes. Dans le fleuve Comoé, ce sont Synodontis bastiani, Synodontis eupterus et Synodontis schall (Mochokidae) qui sont les plus abondantes. La valeur de 0,925 de l'indice de diversité de Simpson témoigne de l'existence d'une grande diversité ichtyologique dans ce fleuve. Les différents paramètres biologiques par espèce $(a, b, K, L \infty)$ révèlent globalement d'une bonne croissance et d'une bonne adaptation des principales espèces d'intérêt commercial dans ces différents cours et retenues d'eau.

(C) 2009 International Formulae Group. All rights reserved.

Mots clés: Diversité ichtyologique, dynamique des populations, bassin de la Comoé, Burkina Faso

\section{INTRODUCTION}

En dehors de quelques travaux d'inventaire partiel (Daget, 1960; Daget et Iltis, 1965; Roman, 1966) aucun inventaire exhaustif de la faune ichtyologique du Burkina Faso et localement, du bassin de la Comoé, n'a été effectué. Plus récemment, le projet Gestion de la Pêche dans le Sud-Ouest (GPSO) a réalisé un travail d'inventaire des espèces, basé pour l'essentiel sur des pêches expérimentales (MEE, 1998). D'autres études, portant sur différents paramètres biologiques, ont été menées sur différents sites disséminés à travers le pays (Baijot et al., 1994; Coulibaly, 2008).

Les études de la diversité piscicole, de la dynamique permettent d'avoir des informations indispensables à la prise de décisions, pour la mise en œuvre des politiques et programmes, pour une gestion durable des ressources halieutiques (Nasser, 1999). En effet, elles permettent d'analyser la structure et la dynamique des populations, d'évaluer la mortalité et d'estimer la production dans le souci de développer des stratégies de gestion (Lévêque et Paugy, 1999; Lorenzo et al., 2002). L'analyse de la relation longueur-poids donne une bonne idée sur l'embonpoint du poisson (Le Cren, 1951).

Deux objectifs principaux sont visés par cette étude à savoir, l'inventaire de la faune ichtyologique et l'analyse de la dynamique des différentes populations, notamment la croissance et l'adaptation aux conditions environnementales des principales espèces d'intérêt commercial. 


\section{MATERIEL ET METHODES \\ Milieu d'étude}

La zone d'étude se trouve dans le bassin de la Comoé, à l'Ouest du Burkina Faso. L'étude a été réalisée sur trois retenues d'eau (Bounouna, Tounoura et Dangouindougou) et sur la partie burkinabè du fleuve Comoé, située dans la Forêt Classée et Réserve Partielle de la Faune de la ComoéLéraba (FCRPF/CL).

\section{La retenue d'eau de Bounouna}

Cette retenue, située au $4^{\circ} 05^{\prime} \mathrm{W}$ et $10^{\circ} 40^{\prime} \mathrm{N}$, est un plan d'eau routier. Elle est construite en 1981 lors du bitumage de la route Bobo-Banfora. D'une profondeur moyenne de $1,5 \mathrm{~m}$ et d'une superficie 2 - 3,5 ha, la retenue peut stocker un volume d'eau variant entre 20000 et $60000 \mathrm{~m}^{3}$. Le sol aux alentours du lac est de type argilo-latéritique. Cette retenue se située dans une zone au climat sud soudanien où alternent deux saisons : une saison des pluies et une saison sèche qui dure 5 à 6 mois (Guinko, 1984). Les précipitations annuelles varient entre 1000 et $1200 \mathrm{~mm}$. La température de l'air est relativement forte $27-28{ }^{\circ} \mathrm{C}$. La retenue est dépourvue de végétation aquatique. Il existe par contre une abondante végétation aux alentours, composée de graminées de diverses espèces ainsi que de quelques espèces fruitières (manguiers essentiellement).

\section{La retenue d'eau de Tounoura}

La retenue d'eau de Tounoura (4 54 ' $\mathrm{W}$ et $10^{\circ} 21^{\prime} \mathrm{N}$ ) a été construite sur le lit d'un cours d'eau lors du bitumage de la route Banfora-Niangoloko en 1983. Sa superficie fluctue entre 6 et 12 ha avec une profondeur moyenne de $2 \mathrm{~m}$. Sa capacité de stockage en eau varie de 30000 à $100000 \mathrm{~m}^{3}$. Le fond est sablo-vaseux. Cette retenue est située dans une zone au climat sud soudanien, avec une saison de pluies et une saison sèche qui dure de 4 à 5 mois (Guinko, 1984). La pluviométrie moyenne annuelle est toujours supérieure à $1000 \mathrm{~mm}$. Il existe une abondante végétation aquatique mais elle n'est pas diversifiée. Les quelques essences recensées autour de la retenue se répartissent en herbacées (Echinochlora colona, Leersia sp, Ceretophyllum submersum, Nymphea sp, Ludwigia sp, Polygonum lamigerium, Oryza barthii, Vorreria filifolia) et en essences ligneuses (Mytragina inermis, Nauclea latifolia, Elaeïs guineensis).

\section{La retenue d'eau de Dangouindougou}

Cette retenue, également artificielle de type routier, située au $5^{\circ} 01^{\prime} \mathrm{W}$ et $10^{\circ} 12^{\prime} \mathrm{N}$, a été construite en 1980. C'est une retenue peu profonde $(0,8-1,2 \mathrm{~m})$ avec une superficie en période de basses eaux de 60 ha et de 75 ha en période de hautes eaux. Elle a une capacité de stockage qui varie de 400000 à $900000 \mathrm{~m}^{3}$. Le bassin versant de cette retenue est particulièrement important avec une superficie de $70 \mathrm{~km}^{2}$. Elle est située dans la même zone climatique que les deux autres. La pluviométrie moyenne est toujours supérieure à $1000 \mathrm{~mm}$ par an. La végétation ligneuse autour de la retenue est très abondante et diversifiée. Il en est de même pour la végétation aquatique. Les berges et surtout les fonds sont couverts d'une végétation dominée par Ceratophyllum sp et Nymphaea sp.

L'ensemble des trois retenues a fait l'objet de nombreuses campagnes d'empoissonnement entre 1989 et 1995, essentiellement avec l'espèce Oreochromis niloticus.

\section{Le fleuve Comoé}

Cours d'eau international, situé dans la zone soudanienne avec une seule saison pluvieuse de 6 à 8 mois et prenant sa source dans les environs de Orodara (Burkina Faso), la Comoé a environ une longueur de $1674 \mathrm{~km}$ dont $514 \mathrm{~km}$ en territoire burkinabè (Traoré, 1999). Son bassin versant couvre une superficie de $17000 \mathrm{~km}^{2}$ et se situe entre $9^{\circ} 35^{\prime}$ et $11^{\circ} 05^{\prime} \mathrm{N}$ et $3^{\circ} 30^{\prime}$ et $5^{\circ} 30^{\prime} \mathrm{W}$. Au Burkina Faso et au Nord de la Côte d'Ivoire, il a un régime tropical de transition caractérisé par une crue unique de juillet à septembre et par une période de basses eaux en mars-avril (Iltis et Lévêque, 1982). Pendant la crue, le débit peut atteindre $500 \mathrm{~m}^{3} / \mathrm{s}$ en septembre ; il redescend à $0,748 \mathrm{~m}^{3} / \mathrm{s}$ en février au moment de l'étiage. Les températures minimales varient entre 24 et $25{ }^{\circ} \mathrm{C}$, et les maximales entre 31 et $32{ }^{\circ} \mathrm{C}$ (Iltis et Lévêque, 1982). Les formations végétales de types savanicoles sont surtout dominées par des espèces comme Isoberlinia doka ou I. dalzielli, Guibourtia coppalifera (Guinko, 1997). On rencontre le long du cours des formations forestières (forêts galeries, forêts denses sèches ; Guinko, 1984). La partie concernée par la présente étude s'étend sur une longueur de $40 \mathrm{~km}$. Les différents sites de collecte sont S1 $\left(09^{\circ} 39^{\prime} \mathrm{N}\right.$ et $\left.04^{\circ} 25^{\prime} \mathrm{W}\right), \mathrm{S} 2\left(09^{\circ} 45^{\prime} \mathrm{N}\right.$ et $\left.04^{\circ} 31^{\prime} \mathrm{W}\right), \mathrm{S} 3$ 
$\left(09^{\circ} 45^{\prime} \mathrm{N} 04^{\circ} 30^{\prime} \mathrm{W}\right), \mathrm{S} 4\left(09^{\circ} 75^{\prime} \mathrm{N}\right.$ et $04^{\circ} 51^{\prime}$ $\mathrm{W}), \mathrm{S} 5\left(09^{\circ} 93^{\prime} \mathrm{N}\right.$ et $\left.04^{\circ} 61^{\prime} \mathrm{W}\right)$.

\section{Collecte de données}

La collecte des données s'est déroulée en deux phases : une première d'août 2003 à avril 2004 sur les trois retenues; une seconde phase de novembre 2005 à avril 2006 sur la portion du fleuve Comoé. La méthode des pêcheries et celle des pêches expérimentales ont été utilisées pour l'échantillonnage. Il a été réalisé tous les mois, à raison de 7 jours de sortie par mois dans les différents milieux d'étude.

Les engins utilisés par les pêcheurs sont composés de nasses, de palangres, de filets épervier et de filets de mailles 20, 22, 37, 42, 50, 65 et $80 \mathrm{~mm}$. Ces données ont ensuite été complétées par des pêches expérimentales avec le filet épervier (maillage $12 \mathrm{~mm}$ ), la senne de plage (maillage $5 \mathrm{~mm}$ ). Ces pêches expérimentales ont permis d'explorer certains milieux des retenues qui ne font pas l'objet de pêche. Ces pêches ont été réalisées avec l'appui des pêcheurs professionnels.

L'identification des poissons s'est faite sur la base des clés d'identification de Paugy et al. (2003).

Deux (2) pesons à ressort de marque SALTER, de portée $1000 \pm 5 \mathrm{~g}$ et $10000 \pm 50$ $\mathrm{g}$, une balance électronique de marque SCALTEC de portée $200 \pm 0,1 \mathrm{~g}$ et un ichtyomètre (gradué au millimètre près) ont été utilisés pour la mesure des paramètres de croissance [poids corporel (P) et longueur totale (LT)]. Les paramètres physicochimiques (pH, température, conductivité) sont mesurés à l'aide d'un appareil combiné de terrain (Combi, marque HANNA).

\section{Traitement des données}

La composition des captures est évaluée au moyen de trois indices que sont :

- la fréquence spécifique (FS) qui représente une fréquence absolue, est le nombre de fois que l'espèce a été rencontrée lors du recensement;

- la contribution spécifique (CS) qui est définie par le rapport de la fréquence spécifique sur la somme des fréquences de toutes les espèces recensées. Il s'agit d'une fréquence relative:
$C S i=\left(F S i / \sum_{i}^{n} F S i\right) * 100$

- l'indice de diversité de Simpson (1949).

$\mathrm{D}=1-\sum_{k=1}^{r i c h} \frac{f k(f k-1)}{n(n-1)}$

$\boldsymbol{D}$ : indice de diversité de Simpson (compris entre 0 et 1), $f_{k}$ : fréquence spécifique de l'espèce dans l'échantillon; $\boldsymbol{n}:$ effectif total de l'échantillon

L'indice de diversité de Simpson a permis de comparer nos résultats avec ceux d'autres études similaires réalisées dans la même zone.

La fréquence d'observation (FO) a été utilisée pour estimer les chances de rencontrer une espèce d'une sortie de pêche à l'autre. Elle a donné des informations sur la présence d'une espèce dans le temps. Elle a été utilisée sur les retenues d'eau vu que la structure des captures des pêcheurs introduisait un biais. Ces derniers camouflaient volontairement une partie des captures (individus de petite taille) pour contourner les services chargés du contrôle des ressources piscicoles au regard de la législation en place. L'indice de diversité n'a donc pas été calculé à ce niveau pour éviter le biais introduit par cette pratique.

\section{Les paramètres a et $b$ des relations longueur - poids}

Les paramètres $\mathrm{a}$ et $\mathrm{b}$ sont déterminés par la forme linéarisée $(\ln \mathrm{P}=\operatorname{lna}+$ blnLT $)$ de la relation $\mathrm{P}=\mathrm{aLT}^{\mathrm{b}}$ (Le Cren, 1951).

LT : Longueur totale; $\mathrm{P}$ : poids corporel; a et $\mathrm{b}=$ constantes.

L'équation de Von Bertalanffy est utilisée pour décrire la croissance des poissons :

$L(t)=L_{\infty} *\left(1-e^{-k(t-t o)}\right)$.

$\mathrm{L}(\mathrm{t})$ : longueur du poisson à l'âge $\mathrm{t}$,

$\mathrm{L}_{\infty}$ : longueur moyenne des poissons très âgés ou longueur asymptotique,

$\mathrm{k}$ : paramètre de courbure (détermine la rapidité du poisson à atteindre $\mathrm{L} \infty$ ),

$\mathrm{t}_{0}$ : âge hypothétique auquel le poisson a une longueur nulle.

Ce modèle envisage la longueur totale en fonction de l'âge. L'analyse des fréquences de longueur est utilisée comme critère d'âge. Pour un meilleur ajustement des courbes de croissance et dans le souci de minimiser les 
irrégularités dans l'estimation de $\mathrm{L}_{\infty}$, nous avons considéré uniquement les six (06) premières années.

\section{Estimation des paramètres de la courbe de croissance de Von Bertalanffy}

Pour le calcul des $\mathrm{k}$ et de $\mathrm{L}_{\infty}$, la méthode graphique de Gulland et Holt (1959) a été retenue pour sa simplicité et surtout sa pertinence car mettant mieux en évidence les observations en conflit avec le modèle de Von Bertalanffy.

L'équation de croissance de Von Bertalanffy inverse a permis l'estimation du paramètre $t_{0}$. Il a toutefois été nécessaire d'exclure les poissons très âgés pour que cette méthode soit applicable c'est-à-dire pour que le terme $\left(1-\mathrm{L}(\mathrm{t}) / \mathrm{L}_{\infty}\right)$ soit toujours positif.

Le test $\Phi$ ' a été utilisé pour comparer les différentes courbes de croissance. Il permet en effet de comparer la croissance de différentes espèces mais aussi celle d'une même espèce vivant dans des milieux différents. Ce test rend compte de la performance de croissance globale (Munro et Pauly, 1983 ; Pauly et Munro, 1984). Cet indice est une valeur chiffrée aisément calculable et se réfère à la seule croissance pondérale si l'on veut comparer des espèces de différentes silhouettes. Il est interprétable biologiquement et présente par ailleurs une variance minimale (Moreau et al., 1986).

$\Phi^{\prime}=\ln \mathrm{k}+2 \ln \mathrm{L}_{\infty}$

La matrice de corrélation est calculée avec le logiciel STATISTICA (version 5.1; 97, Statsoft, France).

\section{RESULTATS}

\section{Paramètres physico-chimiques}

Le suivi des températures moyennes mensuelles (Figure 1A) indique pour l'ensemble des retenues un minimum en décembre (autour de $23{ }^{\circ} \mathrm{C}$ ) et un maximum en avril (autour de $32{ }^{\circ} \mathrm{C}$ ). Les mêmes tendances sont observées dans le fleuve mais l'amplitude thermique est moins importante. Le $\mathrm{pH}$ pour l'ensemble des retenues et le fleuve (Figure 1B) varie peu (entre 7 et 8 en moyenne) et tend à la basicité. La conductivité quant à elle est plus élevée dans les retenues que dans le fleuve (Figure 1C). Elle varie peu au cours de l'année dans l'ensemble des systèmes étudiés sauf dans la retenue de Tounoura où les valeurs sont d'ailleurs les plus élevées.

\section{Faune ichtyologique}

$\mathrm{Au}$ total, 40 espèces regroupées dans 16 familles (Tableau 1) ont été recensées. Les familles des Mormyridae (7 espèces) et des Cichlidae (6 espèces) sont les plus représentées. La richesse spécifique est plus importante dans le fleuve Comoé (38 espèces) que dans les trois retenues (16 espèces). Dans le fleuve Comoé (partie de la Forêt Classée Réserve Partielle Faune de la Comoé Léraba), les espèces recensées sont réparties principalement dans sept (07) familles (Figure 2). Il s'agit de la famille des Cichlidae (6 espèces), des Mormyridae (6 espèces), Cyprinidae (4 espèces), des Claroteidae (3 espèces), des Mochokidae (3 espèces), des Clariidae ( 2 espèces) et des Centropomidae (1 espèce). Parmi ces dernières, la retenue de Dangouindougou (la plus grande en termes de superficie) est celle qui présente le plus grand nombre d'espèces (Tableau 2). Dans ces trois retenues d'eau, ce sont surtout les Cichlidae (Oreochromis niloticus, Tilapia zillii, Sarotherodon galilaeus) qui sont les plus observées, suivies des Osteoglossidae (Heterotis niloticus), des Clariidae (Clarias gariepinus). Quatre (04) espèces dont $H$. niloticus, O. niloticus, $T$. zillii et $C$. gariepinus sont les plus observées dans la pêcherie de Tounoura, avec une prédominance pour les trois (03) premières. A Dangouindougou, il s'agit plutôt des espèces $T$. zillii, S. galilaeus, $H$. niloticus et dans une moindre mesure $C$. gariepinus et Chromidotilapia guntheri qui sont les plus représentées. Toutes les autres espèces, présentes en faible quantité et/ou observées occasionnellement ont été regroupées dans «autres». Il s'agit notamment de Brycinus macrolepidotus, Polypterus endlicheri endlicheri et Schilbe mandibularis. Les Cyprinidae (24\%) et les Mormyridae (18\%) constituent l'essentiel des captures des pêcheurs. La valeur de l'indice de Simpson au niveau du fleuve Comoé est de 0,925, voisine donc de l'unité et témoigne par conséquent de l'importante diversité observée dans cet écosystème. 

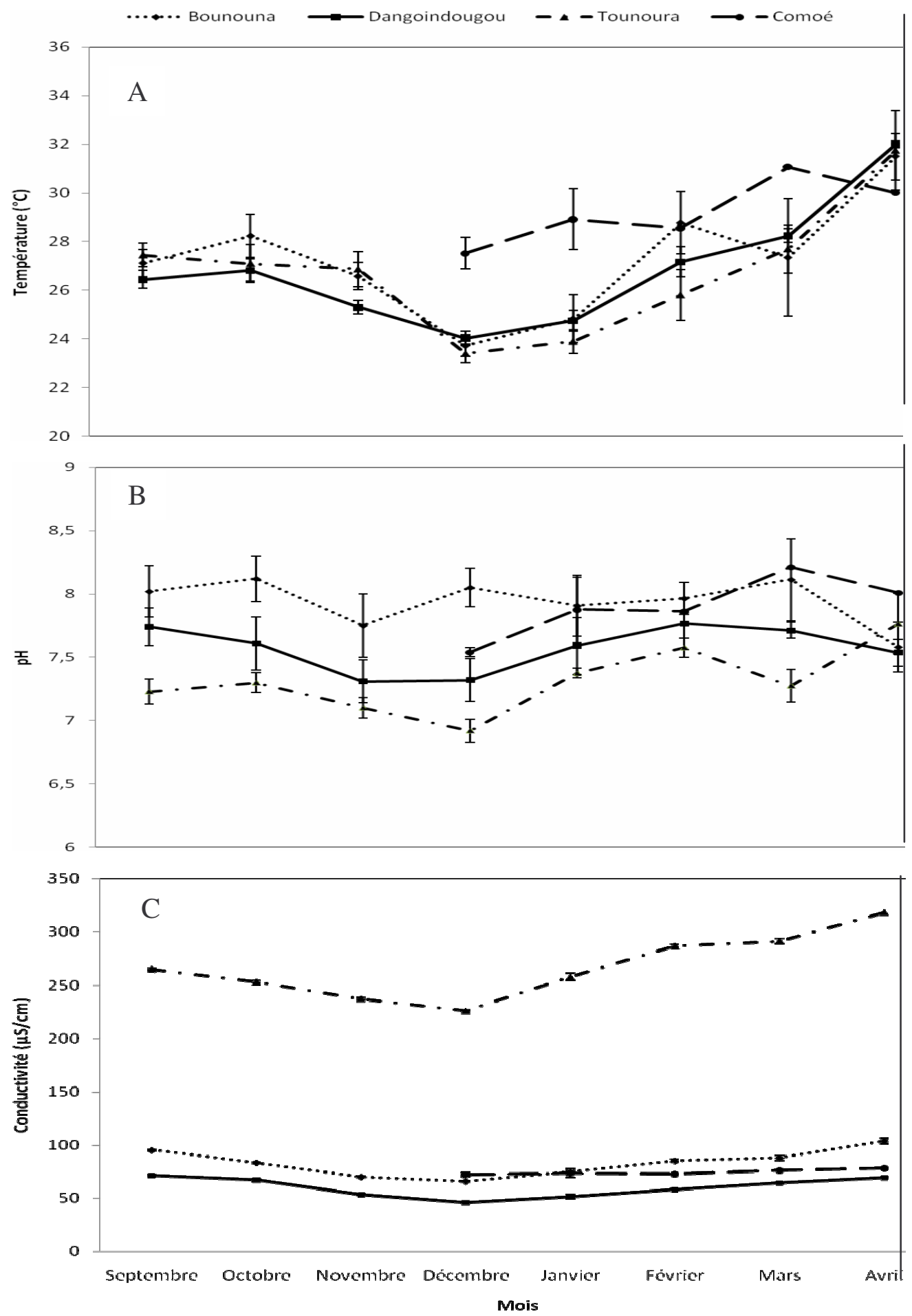

Figures 1 : Evolution de la température (A), du pH (B) et de la conductivité (C) dans les retenues de Bounouna, Dangouindougou et Tounoura dans le bassin de la Comoé (Burkina Faso), de septembre 2003 à avril 2004 et dans le fleuve Comoé de décembre 2005 à avril 2006. Moyenne \pm Ecart type. 
Tableau 1 : Liste des espèces inventoriées dans le fleuve Comoé et les retenues d'eau de Bounouna, Dangouindougou et Tounoura dans le bassin de la Comoé au Burkina Faso d'août 2003 à mars 2004 et novembre 2005 à avril 2006.

\begin{tabular}{|c|c|c|c|c|c|}
\hline \multirow{2}{*}{ Familles } & \multirow{2}{*}{ Noms scientifiques } & \multicolumn{4}{|c|}{ Plans d'eau } \\
\hline & & B & $\mathbf{C}$ & D & $\mathbf{T}$ \\
\hline Polypteridae & Polypterus endlicheri endlicheri Heckel, 184 & & * & & \\
\hline Osteoglossidae & Heterotis niloticus (Cuvier, 1829) & $*$ & $*$ & $*$ & $*$ \\
\hline \multirow[t]{6}{*}{ Mormyridae } & Marcusenius abadii (Boulenger, 1901) & & $*$ & & \\
\hline & Marcusenius senegalensis (Steindachner, 1870) & & $*$ & $*$ & \\
\hline & Mormyrops anguilloides (Linnaeus, 1758) & & $*$ & & \\
\hline & Mormyrus hasselquistii Valenciennes, 1846 & & $*$ & & \\
\hline & Mormyrus rume Valenciennes, 1846 & & * & & \\
\hline & $\begin{array}{l}\text { Petrocephalus bovei (Valenciennes, 1846) } \\
\text { Pollimyrus isidori (Valenciennes, 1846) }\end{array}$ & & $*$ & $*$ & \\
\hline Hepsetidae & Hepsetus odoe (Bloch, 1794) & & $*$ & & \\
\hline \multirow[t]{3}{*}{ Alestidae } & Alestes baremoze (Joannis, 1835) & & $*$ & & \\
\hline & Brycinus macrolepidotus Valenciennes, 1849 & & $*$ & & \\
\hline & $\begin{array}{l}\text { Brycinus nurse (Rüppell, 1832) } \\
\text { Hydrocynus forskali (Cuvier, 1819) }\end{array}$ & * & * & $*$ & * \\
\hline Distichodontidae & Distichodus rostratus Günther, 1864 & & $*$ & & \\
\hline \multirow[t]{5}{*}{ Cyprinidae } & Barbus macrops Boulenger, 1911 & & & * & $*$ \\
\hline & Labeo coubie Rüppell, 1832 & & $*$ & * & $*$ \\
\hline & Labeo parvus Boulenger, 1902 & & $*$ & & \\
\hline & Labeo senegalensis Valenciennes, 1842 & & * & & \\
\hline & Raiamas senegalensis (Steindachner, 1870) & & $*$ & & \\
\hline \multirow[t]{3}{*}{ Claroteidae } & $\begin{array}{l}\text { Auchenoglanis occidentalis (Valenciennes, } \\
\text { 1840) }\end{array}$ & & $*$ & & \\
\hline & Chrysichthys maurus (Valenciennes, 1839) & & $*$ & & \\
\hline & Chrysichthys nigrodigitatus (Lacépède, 1803) & & $*$ & & \\
\hline \multirow[t]{2}{*}{ Schilbeidae } & Schilbe intermedius Rüppell, 1832 & & * & & \\
\hline & Schilbe mandibularis (Günther, 1867) & & $*$ & & \\
\hline Clariidae & $\begin{array}{l}\text { Clarias gariepinus (Burchell, 1822) } \\
\text { Heterobranchus longifilis Valenciennes, } 1840\end{array}$ & $*$ & * & $*$ & $*$ \\
\hline Malapteruridae & Malapterurus electricus (Gmelin, 1789) & & $*$ & & \\
\hline \multirow[t]{3}{*}{ Mochokidae } & Synodontis bastiani Daget, 1948 & & $*$ & & \\
\hline & Synodontis eupterus Boulenger, 1901 & & * & & \\
\hline & Synodontis schall (Bloch et Schneider, 1801) & & $*$ & $*$ & \\
\hline Channidae & Parachanna obscura (Günther, 1861) & & $*$ & * & \\
\hline Centropomidae & Lates niloticus (Linnaeus, 1762) & & * & & \\
\hline \multirow[t]{5}{*}{ Cichlidae } & Chromidotilapia guntheri (Sauvage, 1882) & & $*$ & * & \\
\hline & Hemichromis bimaculatus Gill, 1862 & & * & $*$ & $*$ \\
\hline & Hemichromis fasciatus Peters, 1852 & & $*$ & $*$ & $*$ \\
\hline & Oreochromis niloticus (Linnaeus, 1758) & * & $*$ & $*$ & $*$ \\
\hline & $\begin{array}{l}\text { Sarotherodon galilaeus (Linnaeus, 1758) } \\
\text { Tilapia zillii (Gervais, 1848) }\end{array}$ & $*$ & * & * & $\begin{array}{c}* \\
*\end{array}$ \\
\hline Anabantidae & Ctenopoma kingsleyae Günther, 1896 & & * & * & \\
\hline $\begin{array}{l}\text { Nombre de familles } \\
(\%)\end{array}$ & & $\begin{array}{c}4 \\
(25)\end{array}$ & $\begin{array}{c}16 \\
(100)\end{array}$ & $\begin{array}{c}9 \\
(56)\end{array}$ & $\begin{array}{c}5 \\
(31)\end{array}$ \\
\hline $\begin{array}{l}\text { Nombre d'espèces } \\
(\%)\end{array}$ & & $\begin{array}{c}5 \\
(12,5)\end{array}$ & $\begin{array}{c}38 \\
(95)\end{array}$ & $\begin{array}{l}16 \\
(40)\end{array}$ & $\begin{array}{l}10 \\
(25)\end{array}$ \\
\hline
\end{tabular}

B : Bounouna ; C : Fleuve Comoé ; D : Dangouindougou ; T : Tounoura ; * : Présent. 


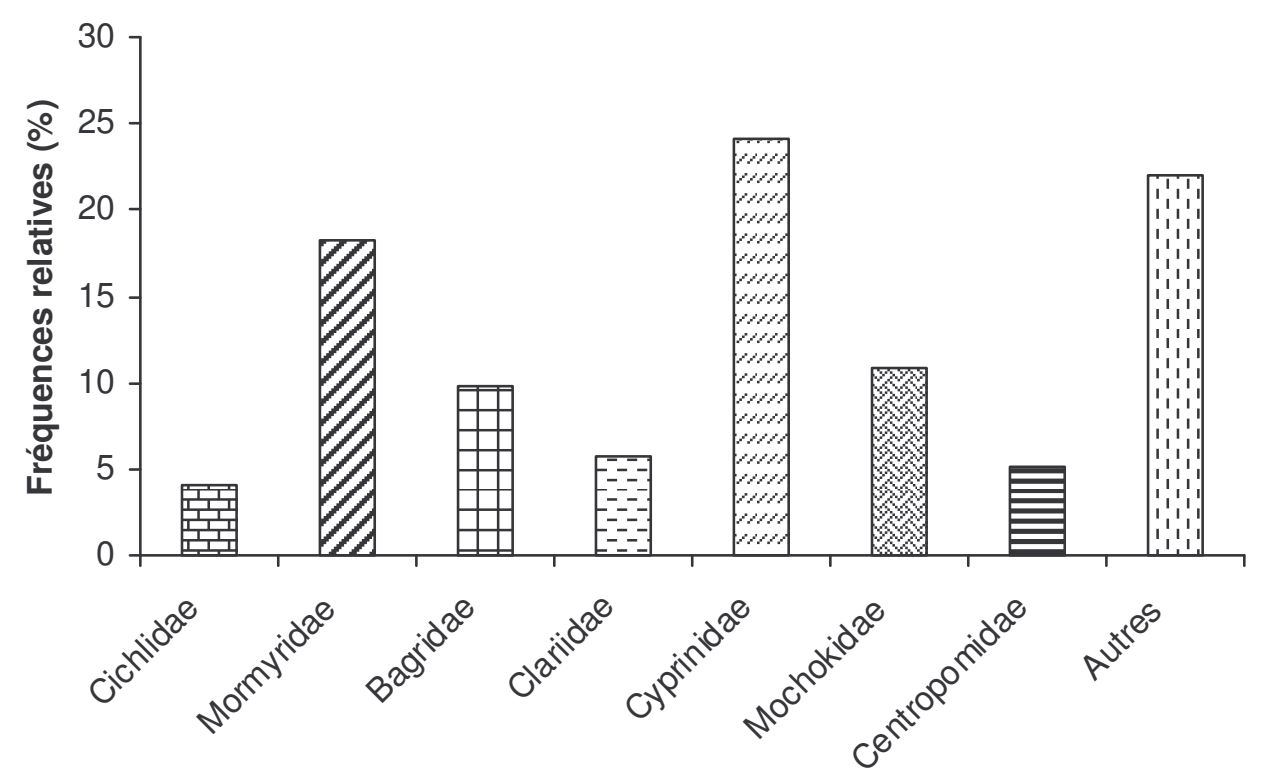

Figure 2 : Importance relative (\%) des différentes familles dans les captures des pêcheurs au niveau du fleuve Comoé dans la Forêt Classée et Réserve Partielle de la Faune de la Comoé-Léraba (FCRPF/CL) au Burkina Faso, de novembre 2005 à avril 2006.

Tableau 2 : Récapitulatif des familles recensées et des espèces rencontrées dans le fleuve Comoé et les retenues d'eau de Bounouna, Dangouindougou et Tounoura dans le bassin de la Comoé au Burkina Faso d'août 2003 à mars 2004 et de novembre 2005 à avril 2006.

\begin{tabular}{|c|c|c|}
\hline Sites & Familles les plus représentées & Espèces les plus représentées \\
\hline Bounouna & Cichlidae & O. niloticus, T. zillii, B. macrops \\
\hline Comoé & Mormyridae, Cichlidae, Alestidae & $\begin{array}{llll}S . \quad \text { schall, } C . & \text { maurus, } & B . \\
\text { macrolepidotus, } & S . & \text { bastiani, } & L . \\
\text { senegalensis, } S . & \text { mandibularis, } & L . \\
\text { coubie }\end{array}$ \\
\hline Dangouindougou & Cichlidae & 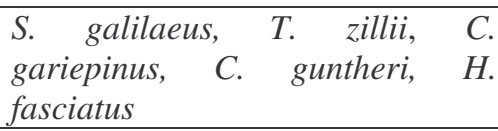 \\
\hline Tounoura & Cichlidae & $\begin{array}{l}\text { O. niloticus, T. zillii, B. macrops, } \\
\text { C. gariepinus }\end{array}$ \\
\hline
\end{tabular}

Sur l'ensemble des espèces observées dans le fleuve Comoé, ce sont celles de la famille des Mochokidae (Synodontis bastiani, Synodontis eupterus et Synodontis schall) $(27,9 \%)$ qui prédominent dans les captures. Des espèces telles que Chrysichthys maurus, B. macrolepidotus, Labeo coubie, Labeo senegalensis et $S$. mandibularis sont également bien représentées (CS varie de 7 à
$10 \%)$. Les espèces qui ont été très peu observées dans les captures des pêcheurs et lors des pêches expérimentales et qui peuvent être considérées comme rares $(\mathrm{CS}<1 \%$ ) sont: Parachanna obscura, O. niloticus, Heterobranchus longifilis, Malapterurus electricus, H. niloticus, Distichodus rostratus, Ctenopoma kingsleyae, C. guntheri, Hemichromis bimaculatus. 
Paramètres de croissance

Paramètres $a$ et $b$ de la relation longueur poids

Les valeurs des paramètres a et $b$ de la relation longueur-poids sont présentées dans le tableau 3. Celles-ci sont comprises entre 0,011 et 0,209 pour a et entre 2,169 et 3,441 pour b. La plus grande valeur de b est obtenue chez O. niloticus dans la retenue de Bounouna et la plus faible chez $H$. niloticus dans la même retenue. Les différentes corrélations sont dans l'ensemble hautement significatives ( $\mathrm{p}<0,001)$.

\section{Paramètres de la courbe de croissance de Von Bertalanffy}

Les méthodes de Gulland et Holt (1959) et l'équation de croissance inverse de Von Bertalanffy ont permis d'estimer les paramètres de la courbe de croissance de Von Bertalanffy (Tableau 4). Les performances de croissance des espèces d'intérêt commercial restent tout de même supérieures à 5. Dans le cas de la retenue d'eau de Bounouna, le manque de données en qualité et en quantité n'a pas permis l'évaluation des paramètres de la courbe de croissance de Von Bertalanffy.

\section{DISCUSSION}

Faune ichtyologique

L'analyse de la diversité à travers l'indice de diversité de Simpson révèle une grande diversité dans le fleuve Comoé, celleci étant néanmoins légèrement inférieure à celle d'une précédente étude réalisée en 2002 sur le même site (données non publiées). La richesse spécifique dans le fleuve Comoé ne semble donc pas avoir évolué dans son ensemble depuis cette période vu que cet inventaire avait recensé 40 espèces avec un indice de diversité de Simpson calculé de 0,928. Toutefois, des modifications sont apparues dans la composition qualitative et quantitative des espèces. $\mathrm{Au}$ cours de l'inventaire en 2002, les espèces les plus abondantes dans les captures étaient $S$. mandibularis, C. maurus, S. schall, B. macrolepidotus contrairement à la présente étude où ce sont plutôt les espèces du genre Labeo qui sont les plus représentées.

Tableau 3 : Paramètres a et $\mathrm{b}$ de la relation longueur-poids de quelques espèces d'intérêt commercial dans le fleuve Comoé et les retenues d'eau de Bounouna, Dangouindougou et Tounoura dans le bassin de la Comoé au Burkina Faso d'août 2003 à mars 2004.

\begin{tabular}{llcccccc}
\hline \multirow{2}{*}{ Retenues } & \multicolumn{1}{c}{ Espèces } & $\begin{array}{c}\text { Classes de tailles } \\
(\mathbf{c m})\end{array}$ & $\begin{array}{c}\text { Effectif } \\
(\mathbf{n})\end{array}$ & $\mathbf{a}$ & $\mathbf{b}$ & $\mathbf{R}^{\mathbf{2}}$ & $\mathbf{p}$ \\
\hline Bounouna & B. macrops & {$[5,7-8,7]$} & 22 & 0,0162 & 3,191 & 0,964 & $<0,001$ \\
& C. gariepinus & {$[21,6-34]$} & 4 & 0,0111 & 2,992 & 0,992 & $<0,001$ \\
& H. niloticus & {$[28-32,2]$} & 5 & 0,2096 & 2,169 & 0,894 & $<0,05$ \\
& O. niloticus & {$[9,4-13,9]$} & 14 & 0,0179 & 3,441 & 0,989 & $<0,001$ \\
& T. zillii & {$[9,2-13,2]$} & 14 & 0,0373 & 3,099 & 0,931 & $<0,001$ \\
\hline Tounoura & B. macrops & {$[7,7-9,5]$} & 27 & 0,0351 & 2,804 & 0,925 & $<0,001$ \\
& C. gariepinus & {$[20,1-61,4]$} & 123 & 0,0257 & 2,751 & 0,937 & $<0,001$ \\
& H. niloticus & {$[22,5-35,6]$} & 524 & 0,0344 & 2,709 & 0,872 & $<0,001$ \\
& O. niloticus & {$[12-29,5]$} & 568 & 0,0376 & 3,077 & 0,965 & $<0,001$ \\
& T. zillii & {$[13,7-26]$} & 286 & 0,0941 & 2,700 & 0,938 & $<0,001$ \\
\hline Dangouindougou & B. macrops & {$[8,7-10,3]$} & 14 & 0,0782 & 2,433 & 0,727 & $<0,001$ \\
& C. gariepinus & {$[15,3-68]$} & 198 & 0,0137 & 2,939 & 0,934 & $<0,001$ \\
& H. niloticus & {$[19,9-82]$} & 569 & 0,0276 & 2,779 & 0,877 & $<0,001$ \\
& O. niloticus & {$[12,7-35,8]$} & 92 & 0,0368 & 3,075 & 0,985 & $<0,001$ \\
& T. zillii & {$[9,9-28,5]$} & 678 & 0,0756 & 2,800 & 0,942 & $<0,001$ \\
\hline
\end{tabular}

a : facteur de condition, constante de la relation longueur-poids ; b : constante de la relation longueur-poids ; p : degré de signification ; $\mathrm{R}^{2}$ : coefficient de détermination. 
Tableau 4: Paramètres de la courbe de croissance de Von Bertalanffy pour les espèces d'intérêt commercial dans le cas des retenues de Tounoura et Dangouindougou dans le bassin de la Comoé au Burkina Faso d'août 2003 à mars 2004.

\begin{tabular}{lccccccccccc}
\hline \multicolumn{1}{c}{ Espèces } & \multicolumn{2}{c}{$\mathbf{L}_{\boldsymbol{\infty}}(\mathbf{c m})$} & \multicolumn{2}{c}{$\mathbf{K}(\mathbf{p a r}$ an) } & \multicolumn{2}{c}{$\mathbf{t}_{\mathbf{0}}$} & \multicolumn{2}{c}{$\boldsymbol{\Phi}$} & \multicolumn{2}{c}{ TMO $(\mathbf{c m})$} \\
\hline & Dan & Tou & Dan & Tou & Dan & Tou & Dan & Tou & Dan & Tou \\
\hline H. niloticus & 38 & 51 & 0,286 & 0,222 & 0,582 & $-2,185$ & 6,023 & 6,359 & 82 & 35,6 \\
O. niloticus & 22 & 33 & 0,500 & 0,769 & 0,632 & 0,134 & 5,489 & 6,730 & 28,2 & 29,5 \\
T. zillii & 31 & 25 & 0,667 & 0,550 & 0,207 & $-0,334$ & 6,463 & 5,840 & 28,5 & 26 \\
\hline
\end{tabular}

Dan : Dangouindougou; Tou : Tounoura $\mathbf{L}_{\infty}$ : longueur asymptotique (longueur moyenne des poissons très âgés); K : paramètre de courbure (détermine la rapidité du poisson à atteindre $L \infty) ; \mathbf{t}_{\mathbf{0}}$ : paramètre de condition initiale (âge hypothétique correspondant à la longueur nulle); $\mathbf{\Phi}$ ': test utilisé pour comparer les différentes courbes de croissance (indice de performance de croissance globale); TMO: Taille Maximale Observée.

S'agissant des retenues, la plus grande diversité observée dans la retenue de Dangouindougou (après celle du fleuve Comoé) pourrait s'expliquer par sa plus grande taille par rapport aux deux autres et par le fait que celle-ci est construite sur un affluent de la rivière Léraba qui se jette par ailleurs dans le fleuve Comoé. En effet, la construction du barrage entre le fleuve et la retenue ne constitue pas une barrière infranchissable à cause du déversoir situé latéralement et qui coule en permanence pendant une bonne partie de l'année. Cette interconnexion peut créer des repeuplements, colonisation ou recolonisation.

La très grande variabilité annuelle et interannuelle du niveau d'eau modifie considérablement l'hydrologie de ces retenues qui se réduisent souvent à des flaques en période d'étiage avec comme conséquence la modification et la disparition des habitats de certaines espèces de poissons. Il s'en suit une raréfaction ou une disparition (parfois momentanée) de certaines espèces inféodées à ces biotopes, faute d'adaptation aux nouvelles conditions écologiques (Xenopoulos et al., 2005). Cette raréfaction ou disparition commence toujours par les espèces les plus sensibles aux variations environnementales, donc les plus vulnérables (Leonardos et al., 2007). Ainsi, deux espèces, Protopterus annectens annectens et Aethiomastabelus nigromarginatus, précédemment recensées parmi les 40 espèces dans le fleuve Comoé (données non publiées), n'ont pas été observées au cours de cette étude. C'est également le cas dans les trois retenues d'eau où il a été constaté l'absence de Schilbe intermedius et Alestes baremoze dans la retenue de Dangouindougou et de Pollimyrus isidori et Hemichromis fasciatus dans les pêcheries de Tounoura et de Bounouna, alors qu'elles avaient été recensées lors des travaux du projet Gestion de la Pêche dans le SudOuest (MEE, 1998). Il faut par ailleurs signaler que la densité humaine a fortement augmenté autour de ces points d'eau (population estimée à Niangoloko à 4252 en 1985 et 8755 en 1996; Nignan et Dembélé, 1997) avec comme corollaire le développement de nombreuses activités anthropiques (maraîchage, fabrication de briques en terre, défrichage des terres pour les cultures), toute chose qui contribue à une dégradation de l'écosystème et partant de la biodiversité comme cela a été décrit par Mc Kinney (2002) et Mallela et al. (2007). Ainsi, cette intense activité humaine autour de ces lacs, a entraîné une eutrophisation importante du milieu et pourrait expliquer les changements observés dans la composition des peuplements. En effet, des travaux antérieurs ont montré que dans des situations de dégradation des écosystèmes, en particulier dans les cas d'eutrophisation, ce sont les espèces les plus tolérantes aux pollutions qui survivent. C'est le cas par exemple des carpes (Cyprinus carpio) que l'on retrouve dans les milieux eutrophes et qui ont été utilisées comme espèces indicatrices de la dégradation de 
l'environnement (Carol et al., 2006). Dans le cas précis de la retenue d'eau de Tounoura, la conductivité a triplé en six (06) ans, passant de $120 \mu \mathrm{S} / \mathrm{cm}$ en 1998 (GPSO, 1999) à 318 $\mu \mathrm{S} / \mathrm{cm}$ en 2004 (figure $1 \mathrm{C}$ ), ce qui témoigne de la présence de fortes teneurs en sels dissouts dans ces milieux. D'ailleurs, il est actuellement observé dans ces retenues, une abondante végétation aquatique sous forme d'îlots flottants qui entrave l'activité de pêche, surtout en période d'harmattan avec le déplacement de celle-ci. Et ce sont les tilapias, espèces très tolérantes vis-à-vis des grandes variations des conditions environnementales qui sont les plus abondantes. Elles pourraient donc servir d'espèce indicatrice. Toutefois, il faut noter qu'une des raisons de l'importance quantitative des Cichlidés dans ces retenues pourraient être les nombreuses campagnes d'empoissonnement menées, au cours desquelles de grandes quantités de tilapia, principalement des individus d'O. niloticus, ont été déversés dans ces retenues. Ces empoissonnements, malgré le fait qu'ils contribuent à amener de nouvelles espèces ou à augmenter quantitativement certaines espèces dans le milieu, entraînent dans de nombreux cas, une perte de la diversité biologique dans ces écosystèmes à travers la fixation de certaines de ces espèces, qui s'adaptent le plus souvent très bien et concurrencent avec les espèces autochtones tant pour l'habitat que pour l'alimentation (Leornardos et al., 2007).

Comparativement aux travaux du projet Gestion de la pêche dans le Sud-Ouest (MEE, 1996), de nouvelles espèces ont été identifiées dans la présente étude. Il s'agit de $S$. galilaeus dans la Comoé, $L$. coubie et $P$. isidori dans la retenue de Dangouindougou et L. coubie, B. nurse respectivement dans celles de Tounoura et Bounouna. Cette apparition ou réapparition de ces espèces peut être révélatrice de conditions écologiques redevenues bonnes, l'année 2003 ayant été particulièrement pluvieuse et donc particulièrement favorable au L. coubie qui est une espèce se reproduisant pendant les crues et précisément dans les zones d'inondation.

\section{Analyse des données de croissance}

Chez la plupart des espèces recensées, la pente de l'équation liant le poids et la longueur (b) révèle une allométrie de croissance. A l'exception de $O$. niloticus qui présente une allométrie de croissance positive (b > 3), les valeurs moyennes chez les autres espèces sont inférieures à 3 , ce qui traduit une allométrie de croissance négative dans l'ensemble de ces réservoirs (Andreu-Soler et al., 2006). Les faibles valeurs $(<2,5)$ observées chez certaines espèces telles que $H$. niloticus à Bounouna et Barbus macrops à Dangouindougou pourraient être liées à l'étroitesse des classes de tailles et du nombre d'individus. Cette situation pourrait être aussi liée à des conditions environnementales défavorables et à la surexploitation des espèces (Torcu-Koc et al., 2006, 2007; Esmaeili et Ebrahimi, 2006). Ces retenues d'eau, fortement anthropisées, sont soumises à la pollution par les intrants agricoles, à une variation très importante du niveau d'eau, ce qui entraîne une forte concentration des éléments dissous qui peuvent se révéler nocifs. Dans la retenue d'eau de Tounoura particulièrement, la pratique du maraîchage sur les berges, couplée aux étiages sévères, ont entraîné une augmentation considérable des valeurs de la conductivité. Or des travaux antérieurs ont montré que les conditions environnementales observées pendant les périodes d'étiage en zone sahélienne affectent négativement la croissance des poissons (Baijot et al., 1994).

$\mathrm{L}_{\infty}$ de $H$. niloticus de la retenue d'eau de Dangouindougou et celui de T. zillii pour celle de Tounoura sont inférieurs à leur taille maximale observée. Cette situation illustre la faible présence des individus de grande taille dans ces plans d'eau (Sparre et Venema, 1996). A l'opposé, $\mathrm{L}_{\infty}$ de T. zillii à Dangouindougou et de $H$. niloticus et $O$. niloticus à Tounoura sont supérieures à leurs tailles maximales observées dans le cas des distributions de tailles moins étalées, ce qui dénote de la longévité relativement faible observée dans ces écosystèmes (Baijot et al., 1994). D'une manière générale, les performances de croissance des espèces sont bonnes dans l'ensemble de ces retenues. Les valeurs de $\Phi$ ' de cette étude sont à ce propos toutes meilleures à celles de certaines retenues d'autres localités du pays (Baijot et al., 1994 ; De Graaf, 2002). En effet, les valeurs de $\Phi$ ' chez $O$. niloticus obtenues par ces auteurs sont de 2,48 dans le barrage de la Tapoa (à l'Est), de 2,47 au Sourou (au Nord), et de 2,32 à 
Ramitenga (au Centre). Par ailleurs, cette espèce avec une croissance isométrique, présente la meilleure croissance, comparativement aux autres espèces. Elle serait donc relativement mieux adaptée aux conditions écologiques de ces retenues.

\section{Conclusion}

Dans le bassin de la Comoé, la faune piscicole est diversifiée. Les Mormirydae et les Mochokidae sont les plus représentées dans le fleuve Comoé; les Cichlidae, les Ostéoglossidae et les Clariidae dans les retenues. La retenue d'eau de Dangouindougou a la plus grande richesse spécifique des trois. La plupart des espèces présentent une bonne adaptation aux conditions environnementales de ces milieux. En général, les performances de croissance des espèces d'intérêt commercial sont supérieures à celles des mêmes espèces sur d'autres retenues d'eau du pays.

\section{REMERCIEMENTS}

Ce travail a été réalisé grâce à un appui financier du Projet BIOTA de la République d'Allemagne.

\section{BIBLIOGRAPHIE}

Andreu-Soler A, Oliva-Paterna FJ, Torralva M. 2006. A review of length-weight relationships of fish from the Segura river basin (SE Iberian Peninsula). J. Appl. Ichthyol., 22: 295-296

Baijot E, Moreau J, Bouda S. 1994. Aspects Hydrologiques et Piscicoles des Retenues d'Eau en Zone Soudano-Sahélienne. Le Cas du Burkina Faso. Centre Technique de Coopération Agricole et Rurale ACP/CEE: Burkina Faso.

Carol J, Benejam L, Alcaraz C, Vila-Gispert A, Zamora L, Navarro E, Armengol J, Garcia-Berthou E. 2006. The effects of limnological features on fish assemblages. Ecology of Freshwater Fish, 15: 66-77.

Coulibaly ND. 2008. Relation taille-poids chez les espèces de poissons de la rivière Sourou au Burkina Faso. Int. J. Biol. Chem. Sci., 2 (3):331-338.

Daget J. 1960. Poissons de la Volta Noire et de la Haute Comoé. Bull. Mus. Natn. Hist. Nat., 32: 320-330.
Daget J, Iltis A. 1965. Poissons de Côte d'Ivoire (eaux douces et saumâtres). Mémoire IFAN, Dakar, p. 385.

De Graaf G. 2002. Fish stock assessment in Comoé, Navrikpé and Tiéfora. In Recueil des Expériences du Projet «Gestion de la Pêche dans le Sud-ouest». GTZ (ed). MAHRH: Bobo-Dioulasso; 237-280.

Esmaeili HR, Ebrahimi M. 2006. Lengthweight relationships of some freshwater fishes of Iran. J. Appl. Ichthyol., 22: 328329.

GPSO. 1999. Rapport des pêches expérimentales, année 1998. Ministère de l'Environnement et de l'Eau, Bobo-Dioulasso, Burkina Faso, p. 52.

Guinko S. 1984. Végétation de Haute-Volta. Thèse de Doctorat ès sciences, Université de Bordeaux III, Bordeaux, p. 318.

Guinko S. 1997. Caractéristiques des unités de végétation et appréciation de la diversité faunique de la zone d'intervention du projet gestion participative des ressources naturelles et de la faune (GEPRENAF). Rapport Ministère de l'Environnement et de l'Eau, Ouagadougou, Burkina Faso, p. 74

Gulland JA, Holt SJ. 1959. Estimation of growth parameters for data at equal time intervals. J. Cons. Ciem., 26 :215-222.

Iltis A, Lévêque C. 1982. Caractéristiques physico-chimiques des rivières de la Côte d'Ivoire. ORSTOM, Paris, 15: 115-130.

Le Cren ED. 1951. The length-weight relationship and seasonal cycle in gonad weight and condition in perca (Perca fluviatilis). Journal of Animal Ecology, 20: 201-219.

Leonardos ID, Kagalou I, Tsoumani M, Economidis PS. 2007. Fish fauna in a protected greek lake: biodiversity, introduced fish species over a 80-year period and their impacts on the ecosystem. Ecology of Freshwater Fish, 17(1): 165-173.

Lévêque C, Paugy D. 1999. Les Poissons des Eaux Continentales Africaines. Diversité, Ecologie, Utilisation par l'Homme. IRD: Paris, France.

Lorenzo JM, Pajuelo JG, Mendez-Villamil M, Coca J, Ramos AG. 2002. Age, growth, reproduction and mortality of the striped seabream, Lithognathus mormyrus (Pisces, Sparidae), off the Canary Islands 
(Central-east Atlantic). J. Appl. Ichthyol., 18: 204-209.

Mallela J, Roberts C, Harrod C, Goldspink CR. 2007. Distributional patterns and community structure of Caribbean coral reef fishes within a river-impacted bay. Journal of Fish Biology, 70: 523-537.

Mc Minney ML. 2002. Do human activities raise species richness? Contrasting patterns in United States plants and fishes. Global Ecology \& Biogeography, 11: 343-348.

Ministère de l'Environnement et de l'eau (MEE). 1996. Rapports enquêtes halieutiques. Résultats des sept sites étudiés : Lobi, Koutoura, Dangouindou, Mare aux Hippopotames, vallée du Kou, Navrikpé et Bounouna. Ministère de l'Environnement et de l'Eau, BoboDioulasso, Burkina Faso, p. 51.

Ministère de l'Environnement et de l'eau (MEE). 1998. Rapport des pêches expérimentales de 1997. Ministère de l'Environnement et de l'Eau, BoboDioulasso, Burkina Faso, p. 52.

Moreau J, Bambino C, Pauly D. 1986. Indices of overal fish growth performance of 100 tilapia (Cichlidae) populations. In The First Asian Fisheries Forum, Maclean JL, Dizon LB, Hosillos LV (eds). Asian Fisheries Society: Manila, Philippines; 201-206.

Munro JL, Pauly D. 1983. A simple method for comparing the growth of fishes and invertebrates. ICLARM Fishsbyte, 1(1): 56.

Nasser AKV. 1999. Length-weight relationships of tuna baitfish from the Lakshadweep Islands, India. Naga, ICLARM Q., 22: 42-44.

Nignan BB, Dembélé O. 1997. Étude socioanthropologique des trames foncières de dix sept (17) villages de la zone d'intervention $d u$ Projet Gestion
Participative des Ressources Naturelles et de la Faune. Geprenaf, Province de la Comoé. Rapport, p. 56.

Pauly D, Munro JL. 1984. Once more on the comparison of growth in fish and invertebrates. ICLARM Fishbyte, 2(1): 21.

Paugy D, Lévêque C, Teugels GG. 2003. Poissons d'Eaux Douces et Saumâtres de l'Afrique de l'Ouest. IRD: Paris, France.

Roman B. 1966. Les Poissons des HautsBassins de la Volta. Musée Royal de l'Afrique Centrale ; Tervuren, Belgique.

Simpson EH. 1949. Measurement of diversity. Nature, 163: 688.

Sparre P, Venema SC. 1996. Introduction à l'évaluation des stocks de poissons tropicaux. Première partie. Document Technique. FAO, 306(1): 1-401.

Torcu-Koc H, Erdogan Z, Treer T. 2006. A review of length-weight relationships of fishes from freshwaters of Turkey. $J$. Appl. Ichthyol., 22: 264-270.

Torcu-Koc H, Erdogan Z, Tinkci M, Treer T. 2007. Age, growth and reproductive characteristics of chub, Leuciscus cephalus (L., 1758) in the _Ikizcetepeler dam lake (Balikesir), Turkey. J. Appl. Ichthyol., 23: 19-24.

Traoré S. 1999. Monographie hydrologique du Bassin Versant de la Comoé à sa confluence avec la Léraba. Rapport Ministère de l'Environnement et de l'Eau, Ouagadougou, Burkina Faso, p. 336.

Xenopoulos MA, Lodge DM, Alcamo J, Maker M, Schulze K, Van Vuuren DP. 2005. Scenarios of freshwater fish extinctions from climate change and water withdrawal. Global Change Biology, 11: 1557-1564. 